\title{
Circulating myeloid-derived suppressor cells are increased and correlate to immune suppression, inflammation and hypoproteinemia in patients with cancer
}

\author{
SHINJI OHKI ${ }^{1}$, MASAHIKO SHIBATA $^{2}$, KENJI GONDA $^{1,3}$, TAKESHI MACHIDA $^{4}$, \\ TATSUO SHIMURA ${ }^{2}$, IZUMI NAKAMURA ${ }^{1}$, TORU OHTAKE ${ }^{1}$, YOSHIHISA KOYAMA ${ }^{1}$, \\ SHINICHI SUZUKI ${ }^{1}$, HITOSHI OHTO ${ }^{3}$ and SEIICHI TAKENOSHITA ${ }^{1}$ \\ Departments of ${ }^{1}$ Organ Regulatory Surgery, ${ }^{2}$ Tumor and Host Bioscience, ${ }^{3}$ Blood Transfusion and \\ Transplantation Immunology, and ${ }^{4}$ Immunology, Fukushima Medical University, \\ 1 Hikarigaoka, Fukushima, Fukushima 960-1295, Japan
}

Received January 31, 2012; Accepted April 3, 2012

DOI: 10.3892/or.2012.1812

\begin{abstract}
Recent studies have identified myeloid-derived suppressor cells (MDSCs) that are potent suppressors of tumor immunity and therefore a significant impediment to cancer immunotherapy. It has been reported that MDSCs are generated by malignant diseases or inflammation. However, no systematic studies in patients have been described. In order to clinically characterize MDSCs, we tested PBMCs from patients with various types of cancer including cholangiocellular, hepatocellular and pancreatic carcinoma, esophageal, gastric and colorectal cancer, breast cancer and thyroid cancer, and GIST, and those from normal volunteers using flow cytometry analysis. A significant increase was seen in the percentages of MDSCs in PBMCs from patients compared with normal volunteers. Among these patients, MDSC level was higher in patients with cancer of the digestive system and patients with breast cancer compared with normal volunteers. MDSC level was significantly and inversely correlated to stimulation indices (SI) of PHA-blastogenesis of lymphocytes and serum concentration of total protein, and positively correlated to neutrophil count. MDSC percentage in patients with gastric and colorectal cancer was also significantly correlated to neutrophil count and inversely correlated with lymphocyte count, and showed highly significant correlation to neutrophil/lymphocyte rate (NLR). In patients with breast cancer, MDSC levels in preoperative patients was significantly increased compared to normal volunteers and significantly decreased in postoperative patients. Thus, it
\end{abstract}

Correspondence to: Dr Masahiko Shibata, Department of Tumor and Host Bioscience, Fukushima Medical University, 1 Hikarigaoka, Fukushima, Fukushima 960-1295, Japan

E-mail: mshibata@fmu.ac.jp

Key words: myeloid-derived suppressor cells, immune suppression, malnutrition, cachexia, breast cancer, digestive system cancer, inflammation is clear that MDSCs are increased in patients with cancer and closely related to suppression of cell-mediated immune responses. These data also suggest that they are related to chronic inflammation and that their levels are increased further in the terminal stages of patients whose nutritional status is impaired as observed in hypoproteinemia. MDSC levels have also been shown to decrease after removal of tumors in patients with breast cancer.

\section{Introduction}

Many cancer immunotherapies developed in experimental animals have been tested in clinical trials. Although some have shown modest clinical effects, most have not been effective $(1,2)$. Recent studies have identified myeloid-origin cells that are potent suppressors of tumor immunity and therefore a significant impediment to cancer immunotherapy. Suppressive myeloid cells were described three decades ago in patients with cancer $(3,4)$, but their functional importance in the immune system has only recently been appreciated (5-8). Indeed, accumulating evidence has now shown that a population of cells with suppressive activity [known as myeloid-derived suppressor cells (MDSCs)] contributes to the negative regulation of immune responses during cancer and other diseases.

MDSCs have been identified in most patients and experimental mice with tumors based upon their ability to suppress $\mathrm{T}$ cell activation. In mice, MDSCs are uniformly characterized by the expression of the cell surface molecule detected by antibodies to Gr1 and CD11b (9). The variation in MDSC phenotype is consistence with the concept that MDSCs are a diverse family of cells that are in various intermediate stages of myeloid cell differentiation (9). In humans, MDSCs are most commonly defined as $\mathrm{CD} 14^{-} \mathrm{CD} 11 \mathrm{~b}^{+}$cells or, more narrowly, as cells that express the common myeloid marker CD33 but lack the expression of markers of mature myeloid and lymphoid cells, and of the MHC class II molecule HLA-DR. In this study, CD11b ${ }^{+} \mathrm{CD} 14{ }^{-} \mathrm{CD} 33^{+}$ cells were analysed as MDSC (10). An accumulation of MDSCs was associated with the decreased number of dendritic cells in the peripheral blood of patients with several types of cancer. 
A
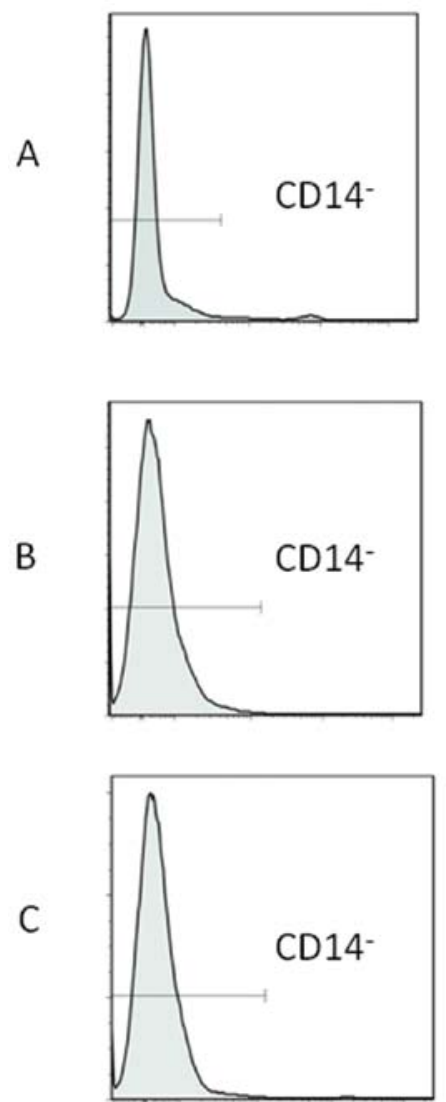
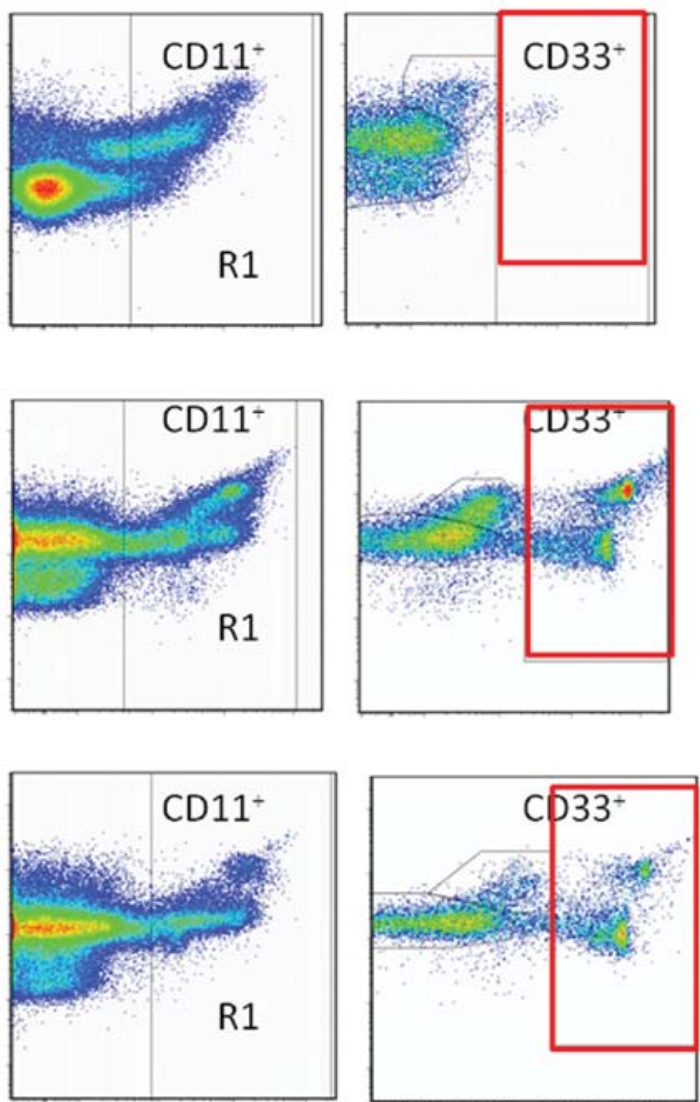

Figure 1. Immunophenotyping of MDSCs by flow cytometry. Cells were labeled with fluorescent isothiocyanate (FITC), phycoerythrin (PE), and Phycoerythrin Cyanin 5.1 (PC5). The cells were incubated with the antibodies for $20 \mathrm{~min}$ at $4^{\circ} \mathrm{C}$ and washed with PBS. These included FITC-conjugated CD14, PE-conjugated CD11b, and PC5-conjugated CD33. (A) Healthy volunteer; (B) a patient with breast cancer; (C) a patient with gastric cancer.

Pathophysiology of MDSC in patients has not been clarified well in contrast to studies in mice.

Tumor development and growth occurs as a result of interactions between the tumor and host immune/inflammatory cells with chronic inflammation having an important role in cancer development and progression (11). Many laboratory markers of systemic inflammatory response, which is closely related to patient's nutritional status, including neutrophil/lymphocyte ratio (NLR) have been investigated as prognostic markers with best evidence for their use demonstrated in surgical patients.

In this report, we show the status of MDSCs in normal volunteers and patients with various types of cancer, and the correlation to laboratory data were analysed.

\section{Materials and methods}

Samples. Blood samples were taken from 53 patients with various types of cancer and 18 normal volunteers with similar age and gender distributions. The patients who received treatment including surgery, chemotherapy, palliative care and follow-up in the Department of Organ-Regulatory Surgery in Fukushima Medical University from January to June, 2011, 45-89 years of age with histologically confirmed cancer were enrolled in the study. Of these 53 patients, 29 had breast cancer, and 21 had cancer of the digestive system including 10 with colorectal, 6 with gastric, 1 with esophageal and 1 with pancreatic cancer, 1 with cholangiocarcinoma, 1 with hepatocellular carcinoma and 1 with GIST, and 3 had thyroid cancer (Table I).
The patients were newly diagnosed as advanced diseases and blood samples were taken before the treatments including surgery and chemotherapy except patients with breast cancer. Peripheral blood mononuclear cells (PBMC) were separated on Ficoll-Hypaque (Pharmacia-Biotech, Uppsala, Sweden). The isolated PBMC were washed twice with RPMI-1640 (Wako Pure Chemical Industries Ltd., Osaka, Japan) and were kept frozen at $-80^{\circ} \mathrm{C}$ until use in freezing media (BLC-1, Juji-Field Co. Ltd., Tokyo, Japan). This study was approved by the ethics committee of Fukushima Medical University (2010-204) and written informed consent was obtained from the patients and normal donors who entered in this study.

Flow cytometry. Cells were labeled for immunofluorescence and analyzed by flow cytometry for cell surface. Cells were labeled with fluorescent isothiocyanate (FITC), phycoerythrin (PE), Phycoerythrin Cyanin 5.1 (PC5). Antibodies were used at 10,10 and $50 \mu \mathrm{g} / \mathrm{ml}$ were diluted each in PBS. The cells were incubated with the antibodies for $20 \mathrm{~min}$ at $4^{\circ} \mathrm{C}$ and washed with PBS. These included FITC-conjugated CD14 (Abcam Cambridge, UK), PE-conjugated CD11b (Beckman Coulter, Marseille, France), PC5-conjugated CD33 (Beckman Coulter). Data acquisition and analysis were performed on a FACSAriaII flow cytometer (BD Bioscience, Mountain View, CA, Fig. 1) using FlowJo software (Tree Star Inc. Ashland, OR).

Proliferation assay. Lymphocyte proliferation assay were carried out with using PBMC suspended in RPMI-1640 (Wako Pure 
Table I. Patients.

\begin{tabular}{lr}
\hline Normal volunteers & 18 \\
Cancer & 53 \\
Digestive system $(\mathrm{n}=21)$ & \\
Cholangiocellular carcinoma & 1 \\
Hepatocellular carcinoma & 1 \\
Pancreatic & 1 \\
Esophageal & 1 \\
Gastric & 6 \\
Colorectal & 10 \\
GIST & 1 \\
Breast & 29 \\
Thyroid & 3 \\
Total & 71
\end{tabular}

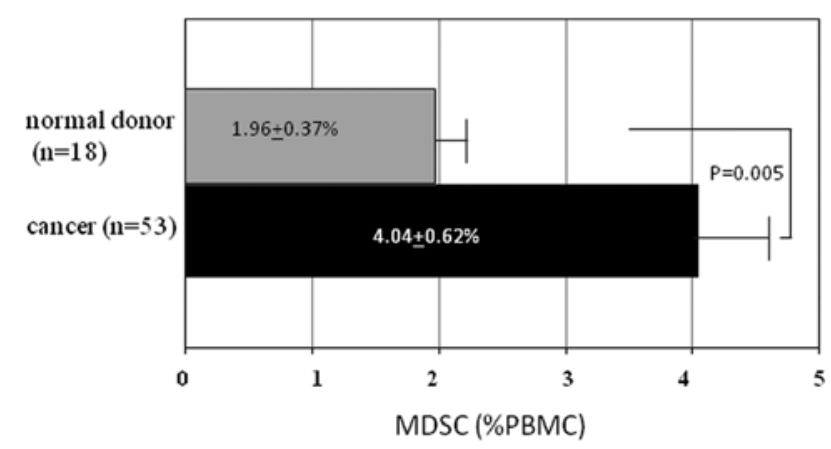

Figure 2. Percentages of circulating MDSC in patients with cancer and in normal volunteers. Percentages of circulating MDSC are shown. Those of 55 patients with cancer was significantly higher than those of 11 normal volunteers $(4.09 \pm 0.62$ vs $1.96 \pm 0.37 \%, \mathrm{p}<0.01)$.

Chemical Industries, Osaka, Japan) and $10 \%$ fetal calf serum (Sigma, St. Louis, MO). Phytohemmaglutinin (PHA) mitogenesis was observed for $80 \mathrm{~h}$ at $10 \mu \mathrm{g} / \mathrm{ml}$ of PHA into PBMC. The cultures were undertaken at $37^{\circ} \mathrm{C}$ in a $5 \% \mathrm{CO}_{2}$ atmosphere with the addition of ${ }^{3} \mathrm{H}$-thymidine (Japan Radioisotope Association, Tokyo, Japan) for the last $8 \mathrm{~h}$ of incubation. Cells were harvested and ${ }^{3} \mathrm{H}$-thymidine incorporation was counted using a liquid scintillation counter (Perkin-Elmer Inc., Waltham, MA) and expressed as count per minute (cpm). Stimulation index (SI) was obtained by calculating total CPM/control cpm in which PHA was not added to PBMC.

Statistical analysis. Differences between the groups were determined by Student's t-test. Relationships between two variables were quantified by Spearman's rank correlation coefficient. Significance was assumed at $\mathrm{p}<0.05$.

\section{Results and Discussion}

We have tested PBMCs from 53 patients with various types of cancer and GIST, and those from 18 normal volunteers. A highly significant increase was seen in percentages of MDSC in

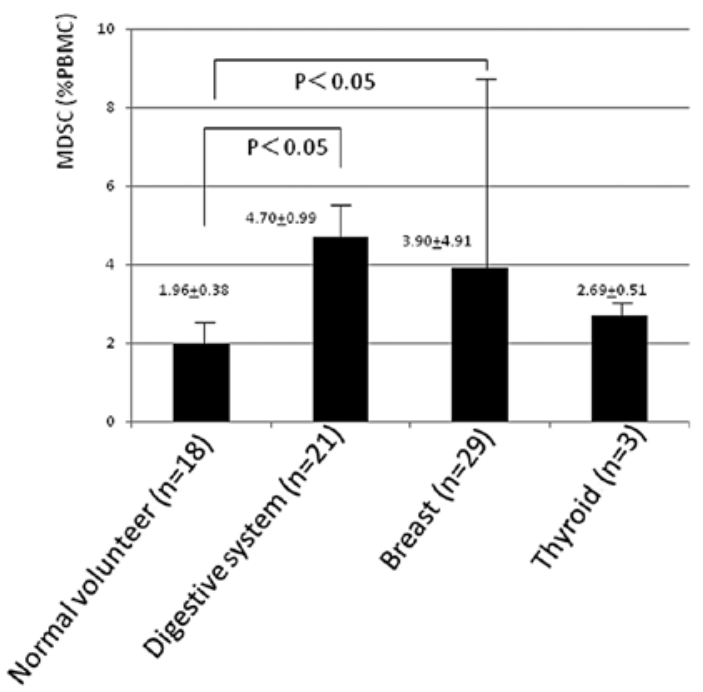

Figure 3. Percentages of circulating MDSCs in patients with cancer and in normal volunteers. Percentages of circulating MDSCs in normal volunteers $(n=18,1.96 \pm 037 \%)$, cancer with digestive system cancer $(n=23$, $4.70 \pm .99 \%)$, with breast cancer $(n=29,3.90 \pm 4.91 \%)$ and with thyroid cancer $(n=3,2.69 \pm 0.51 \%)$ are shown. Those with digestive cancer and with breast cancer were significantly higher than normal volunteers $(\mathrm{p}<0.05$ and $<0.05$, respectively).

PBMCs from patients $(4.04 \pm 0.624 \%, \mathrm{p}<0.005$, Fig. 2) compared with normal volunteers $(1.96 \pm 0.37 \%)$. Among these patients, MDSC (\%) was higher in 21 patients with cancer of digestive system including hepatocellular, cholangiocellular and pancreatic carcinoma, and esophageal, gastric and colorectal cancer, and GIST $(4.70 \pm 0.99, \mathrm{p}<0.05$, Fig. 3) and in 29 patients with breast cancer $(3.90 \pm 4.91, \mathrm{p}<0.05)$ compared with normal volunteers. MDSC was $2.69 \pm 0.51 \%$ in 3 patients with thyroid carcinoma. These data of patients was analysed in correlation to clinical laboratory data and, MDSC (\%) was significantly inversely correlated to stimulation indices of PHA-blastogenesis of lymphocytes $(\mathrm{p}<0.05, \mathrm{r}=-0.271$, Fig. 4A) and serum concentration of total protein $(\mathrm{p}<0.005$, $\mathrm{r}=-0.490$, Fig. 4B), and positively correlated to neutrophil count $(\mathrm{p}<0.005, \mathrm{r}=0.358$, Fig. 4C). MDSC $(\%)$ in detailed digestive system diseases is shown in Fig. 5 and those of 6 patients with gastric cancer and of 10 with colorectal cancer were higher $(\mathrm{p}<0.01$ and $<0.05$, respectively) than in normal volunteers. MDSC (\%) in 16 patients with gastric and colorectal cancer was also significantly correlated to neutrophil count $(\mathrm{p}<0.01, \mathrm{r}=0.477$, Fig. 6A) and inversely with lymphocyte count $(\mathrm{p}<0.05, \mathrm{r}=-0.341$, Fig. $6 \mathrm{~B})$, and showed highly significant correlation to neutrophil/lymphocyte rate $(\mathrm{p}<0.001, \mathrm{r}=0.633$, Fig. 6C). Of the 29 patients with breast cancer, 6 were preoperative and 11 postoperative, MDSC (\%) in preoperative patients was significantly increased compared to normal volunteers $(\mathrm{p}<0.005)$ and it is significantly decreased in postoperative patients $(p<0.05$, Fig. 7) compared to preoperative patients with breast cancer.

The evidence presented in this study shows that percentage of MDSCs in peripheral circulating blood increased in various types of cancer. These percentages inversely correlated to stimulation indices of PHA-blastogenesis, lymphocyte count and serum levels of total protein, and positively to neutrophil count and neutrophil/lymphocyte ratios. Increase of neutro- 
A

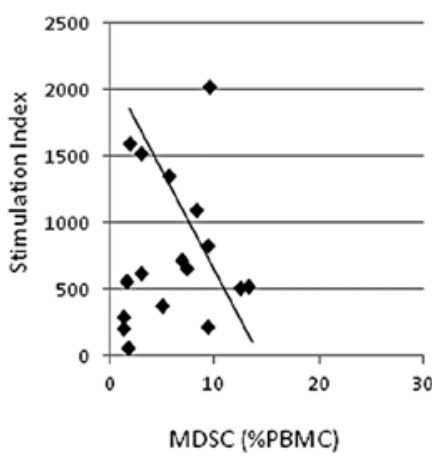

$(p<0.05, r=-0.271)$
B

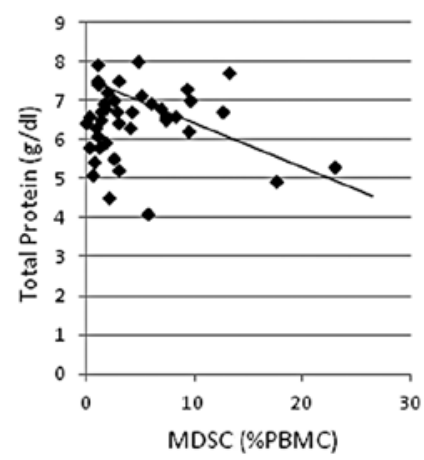

$(p<0.005, r=-0.490)$
C

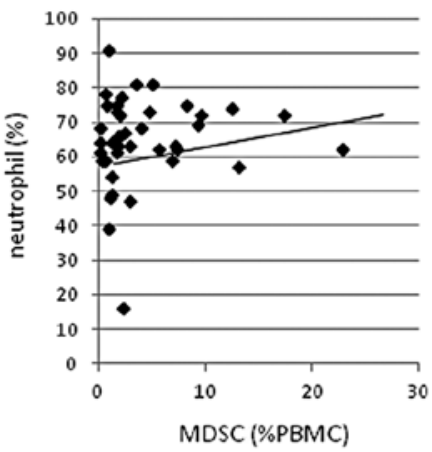

$(p<0.005, r=0.358)$

Figure 4. Correlations of circulating MDSC with PHA blastogenesis of lymphocytes (A), serum levels of total protein (B) and neutrophil counts (C). Percentages of circulating MDSC in 55 patients with cancer are shown. Those significantly correlated to neutrophil counts $(\mathrm{p}<0.005, \mathrm{r}=0.358)$ and, inversely did to stimulation indices of PHA blastogenesis $(\mathrm{p}<0.05, \mathrm{r}=-0.271)$ and serum concentrations of total protein $(\mathrm{p}<0.005, \mathrm{r}=-0.490)$.

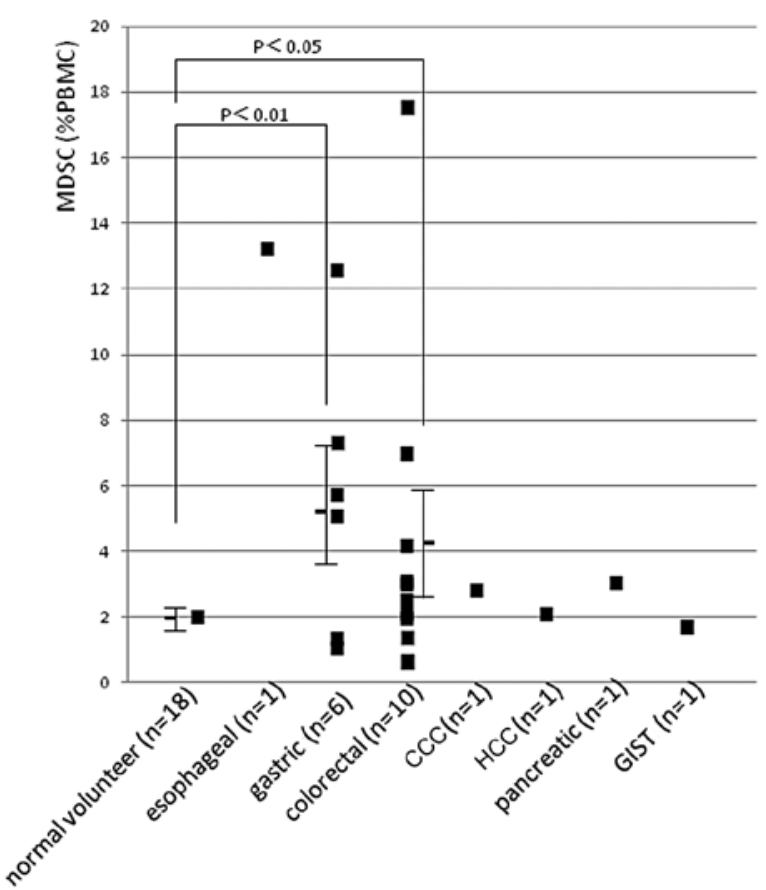

Figure 5. Percentage of circulating MDSCs in patients with cancer of the digestive system and normal volunteers. Percentage of circulating MDSCs in patients with esophageal, gastric and colorectal cancer, and cholangiocellular, hepatocellular and pancreatic carcinoma and GIST are shown. Those with gastric cancer were significantly higher than in normal volunteers $(\mathrm{n}=6$, $\mathrm{p}<0.01$ ) and those with breast cancer were also higher than in normal volunteers $(n=29, p<0.05)$. CCC, cholangiocellular carcinoma; HCC, hepatocellular carcinoma; GIST, gastrointestinal stromal cell tumor.

phil and decrease of lymphocyte are sometimes seen in far advanced patients with malignant diseases in the clinic and the ratios neutrophil/lymphocyte has been used as one of the easiest and effective markers of chronic inflammation and its related immunosuppression in these patients. Thus it is clear that MDSCs are increased in patients with cancer and closely related to suppression of cell-mediated immune responses. These data also suggested that it is increased further in the terminal stages of the patients whose nutritional status is impaired as seen in hypoproteinemia.

In patients with breast cancer, MDSCs (\%) decreased in postoperative condition compared to preoperative patients. MDSCs have been reported to decrease after several kinds of chemotherapy including gemcitabine, 5-FU plus cisplatin (8). On the other hand, it increased by doxorubicin-cyclophosphamide (7) and this may be the results of influence by certain chemotherapeutic agent that induce inflammatory responses. Since some of the postoperative patients received chemotherapy prior to surgery, there may be a possibililty that the decrease of MDSCs seen after the resection of the breast tumor might be a result influenced by chemotherapy. But it seems mainly to be the systemic effect by removal of the tumor since chemotherapy was done approximately 6 weeks prior to the surgery. It is important now to make a further evaluation of the individual effect of chemotherapy or removal of the tumor on changes of MDSCs separately with increased number of patients. If chemotherapy successfully decrease MDSCs, it would be the strong tool as an adjuvant therapy for antigen-specific cancer immunotherapy. The mechanisms of tumor-induced $\mathrm{T}$ cell anergy in patients remains incompletely understood. MDSCs found in the spleen of mice with colon cancer block $\mathrm{T}$ cell function through nitric oxide and arginase production, requiring cell-cell contact (3). Recently the focus has been on highly suppressive myeloid cells infiltrating mouse lung carcinomas, which had high arginase activity and rapidly depleted arginine, blocking T cell proliferation, cytokine production, and $\mathrm{CD} 3 \zeta$ chain expression (4). The exact mechanism of increased production of immature myeloid cells in cancer patients is not clear yet. However, it is known that tumor cells may produce several growth factors and cytokines able to stimulate myelopoiesis $(12,13)$. In addition, vascular endothelial growth factor produced by many tumors is able to affect myelopoiesis (14). It is possible that increased production of these growth factors may affect the normal pathway of cell differentiation resulting in the 
A

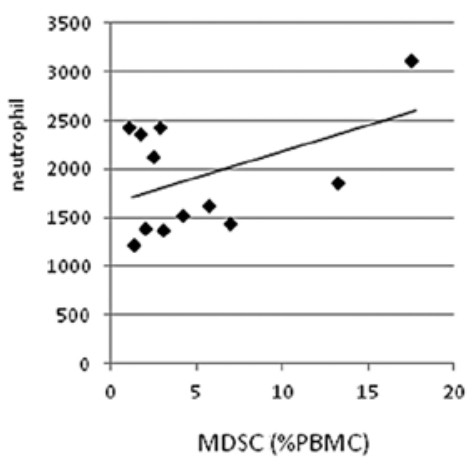

$(p<0.01, r=0.477)$
B

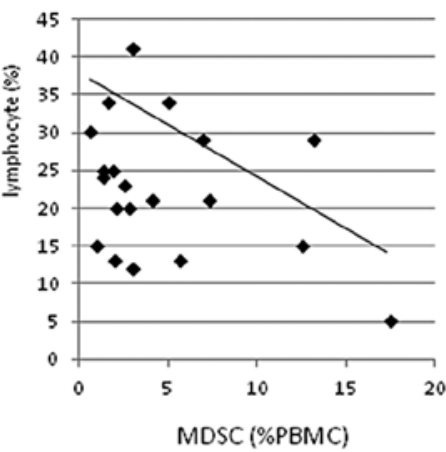

$(p<0.05, r=-0.341)$
C

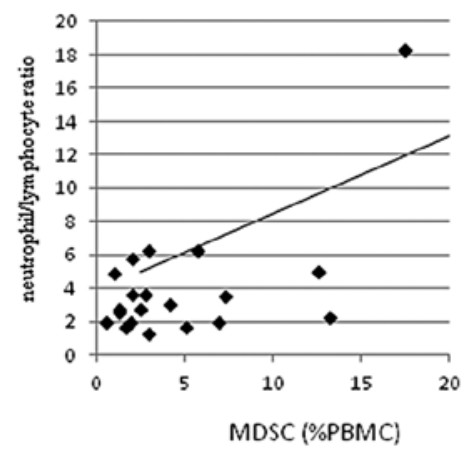

$(p<0.001, r=0.633)$

Figure 6. Correlations of circulating MDSCs with neutrophil counts (A), lymphocyte counts (B) and neutrocytes/lymphocytes ratio (C) in patients with gastric and colorectal cancer. Percentages of circulating MDSCs in 16 patients with gastric and colorectal cancer are shown. Those were significantly correlated to neutrophil counts $(\mathrm{p}<0.01, \mathrm{r}=0.477)$ and neutrophil/lymphocyte counts $(\mathrm{p}<0.001, \mathrm{r}=0.633)$ and inversely with lymphocyte count $(\mathrm{p}<0.05, \mathrm{r}=-0.341)$.

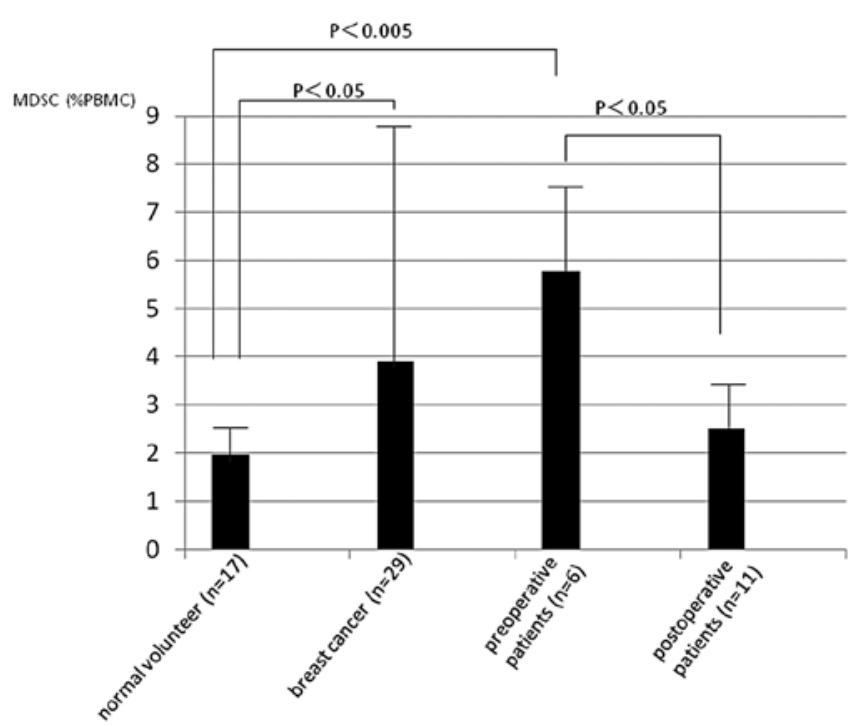

Figure 7. Percentage of circulating MDSCs in patients with breast cancer and normal volunteers. Percentage of circulating MDSCs in 29 patients with cancer are shown. Of these patients, 6 were preoperative and 11 postoperative and others were metastatic and receiving various kinds of chemotherapy. Percentage of MDSCs in the preoperative patients with breast cancer $(n=6$, $5.76 \pm 1.59 \%)$ was significantly higher $(\mathrm{p}<0.005)$ than in normal volunteers and these were decreased in postoperative patients with breast cancer $(\mathrm{n}=11$, $2.50 \pm 0.69 \%, \mathrm{p}<0.05)$. The patients in each group were different and did not overlap.

accumulation of immature myeloid cells. Systemic chronic inflammation has been reported to play a role in developing and growth of tumor and importantly in suppression of tumor immunity $(11,15,16)$. NLR have been reported to be one of the best markers for that and also a good prognostic marker, and to be high in patients with hypoalbuminemia $(17,18)$. In our previous studies, the results showed that a suppression of cell-mediated immune reactions was closely related to the nutritional status, and that it seems to play a role in developing cancer cachexia (19-21).

In conclusion, it was shown that MDSC correlated to nutritional impairment and it may be involved in an immunological mechanism to induce cancer cachexia. It is hoped that we can control immune suppression and chronic inflammation through modulating MDSCs by a selective inhibition by molecular targeting or decreasing with chemotherapy in the near future.

\section{Acknowledgements}

We deeply thank Mr. Shunichi Saito for his technical assistance in preparatory experiments.

\section{References}

1. Rosenberg SA, Yang JC and Restifo NP: Cancer immunotherapy: moving beyond current vaccines. Nat Med 10: 909-915, 2004.

2. Pardoll D and Allison J: Cancer immunotherapy: breaking the barriers to harvest the crop. Nat Med 10: 887-902, 2004.

3. Gabrilovich DI and Nagaj S: Myeloid-derived suppressor cells as regulators of the immune system. Nat Rev Immunol 9: 162-174, 2009.

4. Rosenberg SO and Sinha P: Myeloid-derived suppressor cells: linking inflammation and cancer. J Immunol 182: 4499-4506, 2009.

5. Zea AH, Rodriguez PC, Atkins MB, Hernandez C, Signoretti S, Zabaleta J, McDermott D, Quiceno D, Youmans A, O'Neill A and Ochoa AC: Arginase-producing myeloid suppressor cells in renal cell carcinoma patients: a mechanism of tumor evasion. Cancer Res 65: 3044-3048, 2005.

6. Ochoa AC, Zea AH, Hernandez C and Rodriguez PC: Arginase, prostaglandins, and myeloid-derived suppressor cells in renal cell carcinoma. Clin Cancer Res 13: S721-S726, 2007.

7. Diaz-Montero CM, Salem ML, Nishimura MI, Garett-Mayre E, Cole DJ and Montero AJ: Increased circulating myeloid-derived suppressor cells correlate with clinical cancer stage, metastatic tumor burden, and doxorubicin-cyclophosphamide chemotherapy. Cancer Immunol Immunother 58: 49-59, 2009.

8. Gabitass RF, Annels NE, Crawshaw J, Pandha HS and Middleton GE: Use of gemcitabine-(GEM) and fluoropyrimidinebased chemotherapy to reduce myeloid-derived suppressor cells (MDSCs) in pancreatic (PC) and esophagogastric cancer (EGC). J Clin Oncol 29: 2588, 2011.

9. Kusmartsev S, Nefedova Y, Yoder D and Gabrilovich DI: Antigenspecific inhibition of $\mathrm{CD} 8^{+} \mathrm{T}$ cell response by immature myeloid cells in cancer is mediated by reactive oxygen species. J Immunol 172: 989-999, 2004.

10. Almand B, Clark JI, Nikitina E, van Beynen J, English NR, Knight SC,Carbone DP and Gabrilovich DI: Increased production of immature myeloid cells in cancer patients: a mechanism of immunosuppression in cancer. J Immunol 166: 678-689, 2001. 
11. Blackwill F and Mantovani A: Cancer and inflammation: implications for pharmacology and therapeutics. Clin Pharmacol Ther 87: 401-406, 2010

12. Gabrilovich DI, Chen HL, Girgis KR, Cinninghan HT, Meny GM, Nadaf S, Kavanauch D and Carbone DP: Production of vascular endothelial growth factor by human tumors inhibits the functional maturation of dendritic cells. Nat Med 2: 10961099, 1996.

13. Menetrier-Caux C, Montmain G, Dieu MC, Bain C, Favrot MC, Caux $\mathrm{C}$ and Blay JY: Inhibition of the differentiation of denderitic cells from $\mathrm{CD} 34^{+}$progenitors by tumor cells: role of interleukin- 6 and macrophage-colony-stimulating factor. Blood 92: 4778-4791, 1998.

14. Breitman TR, Collins SJ and Keene BR: Terminal differentiation of human promyelocytic leukemic cells in primary culture in response to retinoic acid. Blood 57: 1000-1011, 1981.

15. Coussens LM and Werb Z: Inflammation and cancer. Nature 420 : 860-867, 2002

16. Forrest LM, McMillan DC, McArdle CS, Angerson WJ and Dunlop DJ: Evaluation of cumulative prognostic scores based on the systemic inflammatory response in patients with inoperable non-small-cell lung cancer. Br J Cancer 89: 1028-1030, 2003.
17. Chua W, Charles KA, Baracos VE and Clarke SJ: Neurrophil/ lymphocyte ratio predicts chemotherapy outcomes in patients with advanced colorectal cancer. Br J Cancer 104: 1288-1295, 2001.

18. Cho H, Hur HW, Kim SW, Kim SH, Kim JH, Kim YT and Lee K: Pretreatment neutrophil to lymphocyte ratio is elevated in epithelial ovarian cancer and predicts survival after treatment. Cancer Immunol Immunother 58: 15-23, 2009.

19. Shibata M, Takekawa M and Amano S: Increased serum concentrations of soluble tumor necrosis factor receptor 1 in noncachectic and cachectic patients with advanced gastric and colorectal cancer. Surg Today 28: 884-888, 1998.

20. Shibata $M$ and Takekawa M: Increased serum concentration of circulating soluble receptor for interleukin-2 and its effect as a prognostic indicator in cachectic patients with gastric and colorectal cancer. Oncology 56: 54-58, 1999.

21. Shibata M, Nagata $Y$, Kimura $T$, Kanou H, Nezu T and Fukuzawa M: Elevated serum concentration of interleukin-1 receptor antagonist (IL-1ra) is correlated to interleukin-6 and to hypoalbuminemia in cachectic patients with colorectal cancer. Int J Clin Oncol 5: 116-120, 2000. 